\title{
Computer depot repair service
}

\author{
GEORGE HAYASHIDA \\ Three Delta Corporation, Sunnyvale, California 94086
}

\begin{abstract}
The services of computer repair depots are noted and the special services of Three Delta Corporation are described.
\end{abstract}

Ever since the wheel was invented, there was and still is a need for some type of repair service. Computers are no different. They stop, hiccup, and so on, due to normal wear and tear. The user asks why? But why does a light bulb go "out," especially when you feel you need it most?

We do not always know why these happenings occur, but they do. Statistics show that approximately $10 \%$ of the electronic units, whether they are computers, $\mathrm{I} / \mathrm{O}$ devices, or whatever, fail sometime during a given year. A user's system may fail this minute, this year, next year, or in 5 years. Who knows? If and when it does, a depot repair service can provide the user with the maximum return for the money.

Several types of services and their advantages and disadvantages are listed below.

\section{Depot Service (Three Delta Corporation)}

Minimum cost (only repair what is needed), but the user must send bad unit to depot for repairs. The customer must have some expertise in diagnosing problems. And the user must have spares if he/she cannot wait for the normal turnaround time (10 working days). Special expedite service $(72 \mathrm{~h})$ is available for extra fee.

\section{On-Call Service}

Medium expense-customer pays by the hour for services rendered. In most cases, minimum charges are for $2 \mathrm{~h}$ plus mileage, to and from, and a percentage of the price of a new board for the replacement unit. This can become very expensive if a memory management unit (DEC M7236) is defective. In many cases, service groups may be unable to repair memories unless they are the OEM group that is offering this service.

Requests for reprints and additional information should be addressed to the author c/o Three Delta Corporation, $1038 \mathrm{Kiel}$ Court, Sunnyvale, California 94086.

\section{Yearly Contract Service}

This is the most expensive service and is generally offered by OEM group. This is an insurance-type service where you continually pay for service that may not be needed for a long time. This service may offer the fastest turnaround time if the service group is located close to your facility.

\section{Mailer Service (DEC)}

Moderate cost per unit but very expensive to start. This is a prepaid yearly program. The user contracts for $\mathrm{X}$ amount of dollars of repairs for the year and deposits that amount at the start of the program.

\section{SUMMARY}

The depot repair service can fit most of the users' needs. Three Delta Corporation, since its inception in early 1972 , has been dedicated to providing the best possible service to the data processing and computer industries by offering a repair capability second to none.

Three Delta Corporation's expertise in repair, test, and refurbishment of computer memories and logic cards has filled the gap that exists between the original manufacturer and the equipment user. It is unfortunate, but too often true, that OEMs are not motivated to provide the end user with the type of service required to keep down-time to a minimum and equipment utilization to a maximum. This results in unnecessary expenditures of time and money by creating delays that are unacceptable.

Three Delta Corporation offers these (and repairs of all types) of printed wiring boards, core memory systems and stacks, semiconductor memories, and computer logic boards. Our experience-over 20,000 units successfully repaired, along with quick turnaround, quality workmanship, and 90-day warranty-can help eliminate these costly delays. Service is Three Delta's only product. 